\title{
The Effect of Decoherence on the Contextual and Nonlocal Properties of a Biphoton
}

\author{
A. Shaham and H. S. Eisenberg
}

\author{
Racah Institute of Physics, Hebrew University of Jerusalem, Jerusalem 91904, Israel
}

\begin{abstract}
Quantum contextuality is a nonintuitive property of quantum mechanics, that distinguishes it from any classical theory. A complementary quantum property is quantum nonlocality, which is an essential resource for many quantum information tasks. Here we experimentally study the contextual and nonlocal properties of polarization biphotons. First, we investigate the ability of the biphotons to exhibit contextuality by testing the violation of the KCBS inequality. In order to do so, we used the original protocol suggested in the KCBS paper, and adjusted it to the real scenario, where some of the biphotons are distinguishable. Second, we transmitted the biphotons through different unital channels with controlled amount of noise. We measured the decohered output states, and demonstrated that the ability to exhibit quantum contextuality using the KCBS inequality is more fragile to noise than the ability to exhibit nonlocality.

PACS numbers: 03.65.Yz, 03.65.Ud, 42.50.Xa, 42.50.Lc
\end{abstract}

Quantum theory has a unique probabilistic nature, different from any classical theory. It is manifested by a surprising behavior that doesn't have any classical analogue: while measurements performed on classical systems have predefined outcomes, compatible with a non-contextual hidden variable assumption, quantum systems are contextual - the measurement outcome of a quantum system does depend on the measurement arrangement and the choice of observables. This contradiction between quantum mechanics predictions and non-contextual hidden variable models, is manifested in the Kochen-Specker theorem [1], and can be tested using quantum systems with a Hilbert space dimension of $d \geq 3[1,2]$. A particular example for this conflict is the famous EinsteinPodolsky-Rosen (EPR) paradox [3] : when measurements are performed on two spatially separated quantum subsystems, one may observe nonlocal correlations which can not be reproduced by any local hidden variable model.

In 1964, John Bell proposed an experimental test in which one can refute the existence of local hidden variables, by violating an inequality, which is satisfied by classical theories [4]. Later on, the CHSH inequality a simpler version of Bell inequality, which is suitable for two-qubit entangled states, was derived [5]. This inequality has been tested experimentally, favoring the nonlocal predictions of quantum mechanics [6]. With the growing interest in quantum information, it was discovered that states that can violate Bell inequalities are important for many quantum processing techniques such as quantum teleportation and quantum cryptography [7]. The Horodecki criterion for the optimal observables for the CHSH operator should be used in order to evaluate whether a given state can violate the CHSH inequality and thus, serve as a resource for such tasks [8].

In his response to the EPR paper, Bohr pointed out that EPR-like peculiarities can be observed without a spatial separation between the system components 9]. This was rigorously proved by Kochen and Specker [1].
Later on, proposals for experiments (i.e., the manifestation of non-contextual inequalities) that are aimed to distinguish between quantum mechanical predictions and non-contextual realism have been suggested $10-12$. A special focus was on the quantum features of threelevel systems (qutrits), which are the simplest systems that can exhibit contextual correlations, but not nonlocal ones. Inconsistency between quantum predictions for measurements performed on qutrits and classical assumptions has already been described by Wright [13]. More recently, a contextual inequality which is suitable for qutrits was derived by Klyachko, Can, Binicioğlu, and Shumovsky (KCBS) 14]. This inequality is state dependent, and was proved to be the simplest contextual inequality. In its geometrical form, the KCBS inequality resembles Wright inconsistency, giving it a contextual interpretation. So far, contextual inequalities were tested experimentally using several realizations of quantum systems including photonic systems [15 17], neutrons [18], trapped ions [19], and nuclear spins [20]. Specifically, a violation of the KCBS inequality using indivisible photonic qutrits, encoded in the spatial and polarization degrees of freedom, was reported in Ref. 21. A KCBS violation was also used to certify the randomness of a random number generator, based on the measurements of a single trapped ion qutrit 22]. Recently, Ahrens et al. reported on two experiments where in the first one, Wright inconsistency was demonstrated using multimode single-photon qutrits [23]. In the second one, the same qutrit source was used to achieve a KCBS violation with the minimal required number of projections. A violation of the geometrical form of the KCBS inequality has also been reported by Kong et al. who implemented the qutrits using NV centers [24].

In this work, we investigated the contextual and nonlocal properties of biphotons - pairs of photons that occupy the same spatio-temporal mode [25]. We encoded the information in the polarization degree of freedom. Due to 
the symmetry of such state, it is confined to the twoqubit triplet subspace. One option to span this subspace is with three out of the four maximally entangled Bell states, when the singlet is omitted. Thus, the same system can exhibit either contextual properties, or nonlocal properties when the two photons are divided. Recently, Soeda et al. suggested a simple hierarchy between contextuality and nonlocality [26]. They showed that contextuality with respect to the KCBS inequality implies nonlocality, when the two-photons are separated, while the opposite is not true.

In the first part of this letter, we use the projection protocol suggested by Klyachko et al. 14 to test the biphoton's ability to violate the KCBS inequality in its geometric representation. We find that distinguishability imperfections of the biphoton [27] dramatically affect the measured KCBS value. Thus, it is important to quantify these imperfections in order to faithfully demonstrate contextuality. In the second part, we explore the effects of decoherence on the biphoton ability to exhibit different aspects of quantumness. The biphotons have passed through three major examples of controlled unital channels [28, 29], and the biphoton output state was characterized by a quantum state tomography procedure. We study the nonlocal and the contextual properties of partially polarized biphotons, and their relative hierarchy.

A general qutrit wave function of a biphoton state $|\psi\rangle$ can be written as

$$
|\psi\rangle=\alpha_{0}|2,0\rangle+\alpha_{1}|1,1\rangle+\alpha_{2}|0,2\rangle,
$$

where $\left|n_{h}, n_{v}\right\rangle$ represents a two-photon state that is composed of $n_{h}$ horizontally polarized photons and $n_{v}$ vertically polarized photons. A qutrit state is defined as neutrally polarized if its spin projection onto the $\mathrm{Z}$ axis is zero. A common qutrit basis of neutrally polarized states is composed of the following states 25]: $\left\{\left|\psi_{h v}\right\rangle \equiv|1,1\rangle,\left|\psi_{p m}\right\rangle \equiv(|2,0\rangle-|0,2\rangle) / \sqrt{2},\left|\psi_{r l}\right\rangle \equiv\right.$ $(|2,0\rangle+|0,2\rangle) / \sqrt{2}\}$. [14]

Consider the geometric form of the KCBS inequality

$$
K=\sum_{k=1}^{5}\left|\left\langle l_{k} \mid \psi\right\rangle\right|^{2} \leq 2,
$$

where $\left|l_{k}\right\rangle$ are five qutrit states that satisfy $\left|l_{k}\right\rangle \perp\left|l_{k+1}\right\rangle$ $(k+1$ modulo 5$)$, and $|\psi\rangle$ is the qutrit state of interest. Since this inequality is state-dependent, different $|\psi\rangle$ states require different sets of $\left|l_{k}\right\rangle$ to achieve maximal $K$ values. There are some qutrit states, like the $|2,0\rangle$ state, that can not exhibit violation for any chosen set of $\left|l_{k}\right\rangle$. It can be shown that by choosing $|\psi\rangle$ to be neutrally polarized, and by choosing a quintuplet of $\left|l_{k}\right\rangle$ such that $\left|\left\langle l_{i} \mid \psi\right\rangle\right|=\left|\left\langle l_{j} \mid \psi\right\rangle\right|$ for any $i$ and $j$, a maximal violation of Eq. (2) is obtained, where the KCBS value is $K=\sqrt{5} \approx 2.24>2$.
Given a qutrit state, one can measure its KCBS value either by reconstructing the whole density matrix of the state and then calculating the projection value on each $\left|l_{k}\right\rangle$, or by performing direct projection measurements on each $\left|l_{k}\right\rangle$ state. According to the projection measurement protocol for biphoton qutrits suggested by Klyachko et al. 14], one should choose the $\left|l_{k}\right\rangle$ states to be neutrally polarized. Thus, it is possible to write every biphoton state $\left|l_{k}\right\rangle$ as a superposition of two orthogonally polarized single photons $|s\rangle$ and $|t\rangle$

$$
\left|l_{k}\right\rangle \equiv\left|l_{s t, k}\right\rangle=\frac{\left|s_{k}\right\rangle\left|t_{k}\right\rangle+\left|t_{k}\right\rangle\left|s_{k}\right\rangle}{\sqrt{2}} .
$$

With the usage of a Hanbury Brown-Twiss interferometer (i.e., separating the biphoton into two arms $\{1,2\}$ by a beam splitter (BS)), one can project the biphoton onto the orthogonal separable polarization state $|s\rangle_{1}|t\rangle_{2}$. Assuming that the generated biphoton state occupies only the qutrit symmetric subspace, and an ideal projection setup, the coincidence count rate for the latter projection should be equal to the rate of projection onto the $|t\rangle_{1}|s\rangle_{2}$ state, and their sum to the biphoton projection rate onto the $\left|l_{s t, k}\right\rangle$ state.

In practice, distinguishability between the two biphoton photons has to be considered. As was shown by Adamson et al. [27], a realistic biphoton state is not perfect and may contain some photon-pairs in the antisymmetric singlet subspace. Such unwanted photon pairs will be successfully projected onto every pair of orthogonal polarizations $|s\rangle$ and $|t\rangle$. As a result, the KCBS value calculated directly from the projection measurements can even be higher than $\sqrt{5}$, which is the upper limit allowed by quantum mechanics. Furthermore, although the singlet subspace should be incoherent with the qutrit subspace [27], imperfect symmetry between the two arms of the projection setup may result in partially coherent terms between the two subspaces. The appearance of these coherent terms results in different coincidence rates for the $|s\rangle_{1}|t\rangle_{2}$ and $|t\rangle_{1}|s\rangle_{2}$ projections, and they deviate equally from bellow and above the rate that corresponds to the ideal biphoton state. Therefore, with the direct projection method, the coincidence rate $N_{s_{k}, t_{k}}$ should be considered as the average of the $\left|s_{k}\right\rangle_{1}\left|t_{k}\right\rangle_{2}$ and $\left|t_{k}\right\rangle_{1}\left|s_{k}\right\rangle_{2}$ rates in order to eliminate the artificial coherence effect. Additionally, the relative part of the generated singlet states should be subtracted from each coincidence rate $N_{s_{k}, t_{k}}$. For that end, the singlet content should be estimated using other projection measurements. Two possible ways can be either by separating the biphoton to two different spatial modes and measuring the visibility of a Hong-Ou-Mandel interference [30], or by performing a complete two-photon four dimensional (4D) quantum state tomography (QST) procedure.

In order to measure the KCBS value of biphotons, we generated $|1,1\rangle$ states and performed the projection protocol described above. The experimental setup is shown 


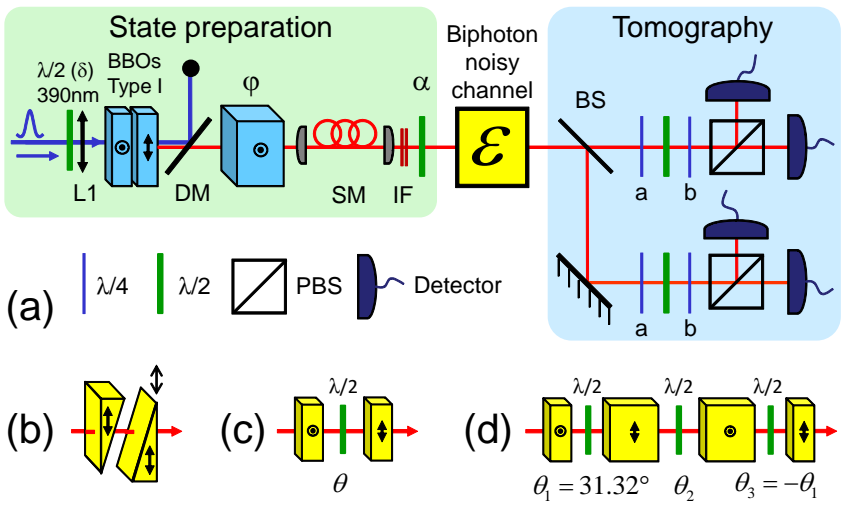

FIG. 1. (Color online) The experimental setup. (a) Biphoton generation and characterization units: photon pairs are generated in the BBO crystals, which are located after a lens (L1) and a half-wave plate (HWP, $\lambda / 2$ ) whose angle is $\delta$. The down-converted photon-pairs pass through a dichroic mirror (DM), a birefringent compensating crystal $(\varphi)$, a single-mode fiber (SM), an interference bandpass filter (IF), and another HWP $(\alpha)$. In the state characterization unit, the biphotons are split probabilistically by a beam splitter (BS) into two ports. In each port, the photons pass a sequence of a quarterwave plate (QWP, $\lambda / 4, \mathrm{a})$, a HWP, another QWP (b) and a polarizing beam splitter (PBS) before being coupled into single-photon detectors. The decoherence channels $(\mathcal{E})$ are plugged in only when the noise affects on the biphotons are studied. (b) The one-field (dephasing) channel, composed of two translatable quartz wedges. (c) The two-field channel, composed of two perpendicularly oriented identical $2 \mathrm{~mm}$ thick calcite crystals. (d) The three-field (isotropic) channel. This channel is composed of four crystals and two fixed HWP. The thickness of the two outer (inner) crystals is $1 \mathrm{~mm}$ $(2 \mathrm{~mm})$. The fields' strength of both the two- and the threefield channels is set by rotating the middle HWP.

in Fig. 1a (see elaboration in the supplementary material [31]). A pulsed laser pumps two perpendicular nonlinear crystals of equal length [32], and generates biphotons in the process of spontaneous parametric down conversion. The generated biphotons are in the state of $|\psi\rangle=\cos (2 \delta)|2,0\rangle+\sin (2 \delta) e^{i \varphi}|0,2\rangle$, where $\delta$ is the angle of a half-wave plate (HWP) that controls the pump beam polarization. Tilting a birefringent crystal that is placed after the generating crystals for temporal compensation controls the angle $\varphi$. Setting $\delta=22.5^{\circ}$ and $\varphi=180^{\circ}$ results in a generation of a neutrally polarized $\left|\psi_{p m}\right\rangle$ state. The $\left|\psi_{h v}\right\rangle(|1,1\rangle)$ state is generated by passing the $\left|\psi_{p m}\right\rangle$ state through another HWP oriented in an angle of $\alpha=22.5^{\circ}$.

In the characterization unit, the biphoton is probabilistically split using a BS into two ports. Then, the two-port polarization state is projected onto a separable two-qubit polarization state. Thus we are able to perform the projection protocol, or alternatively, to characterize the two-photon state using a complete 4D QST procedure 33.

We generated a $|1,1\rangle$ biphoton state and reconstructed its two-photon four-dimensional density matrix $\hat{\rho}$. We obtained a fidelity of $93 \pm 1 \%$ to the ideal state, where $99 \pm 1 \%$ of the state population occupied the symmetric subspace.

A KCBS value of $K=2.16 \pm 0.02>2$ is calculated from the measured two-photon $4 \mathrm{D}$ density matrix $\hat{\rho}$, demonstrating violation of $8 \sigma$. The calculation was carried out by projecting the symmetric subspace of $\hat{\rho}$ on a $\left|l_{k}\right\rangle$ quintuplet (see elaboration on the $\left|l_{k}\right\rangle$ states in the supplementary material [31]). Errors were calculated using Monte-Carlo simulations assuming Poisson distribution for the counts [34]. Performing the direct projection measurements, we measured a violation of $4 \sigma$, with a KCBS value of $K=2.17 \pm 0.04$. The singlet population which is required for the direct projection protocol was taken from the measured $4 \mathrm{D}$ density matrix of the biphoton. Here, the error value was estimated assuming Poisson distribution for the projections onto the $\left|l_{k}\right\rangle$ states, and by performing Monte-Carlo simulations to derive the error for the singlet subspace population.

In order to compare the resilience of two fundamental characters of quantum mechanics, contextuality and nonlocality, we explore the effects of decoherence on the ability of biphotons to exhibit these nonclassical effects. Neutrally polarized biphotons were transmitted through different types of quantum noisy channels and the resulting two-photon 4D density matrices were reconstructed. Specifically, we compered between the final states ability to post-selectively violate the CHSH nonlocality inequality and its ability to demonstrate contextuality by violating the KCBS inequality (2). The CHSH operator $S_{C H S H}$ is a function of $\hat{\rho}$ obtained by four different projection measurements, and nonlocality is demonstrated when the inequality $\left|\left\langle S_{C H S H}\right\rangle\right| \leq 2$ is violated [5]. For a measured output state $\hat{\rho}$, the maximal value of $\left|\left\langle S_{C H S H}\right\rangle\right|$ was calculated using the Horodecki criterion [8], and the maximal KCBS value was obtained using a numerical search for the $\left|l_{k}\right\rangle$ quintuplet that gives the highest KCBS value.

The operation $\mathcal{E}$ of a quantum channel on a state $\hat{\rho}$ is usually defined by a map $\hat{\rho}^{\prime}=\mathcal{E}(\hat{\rho})$, where a unital channel satisfies $\mathcal{E}(\hat{I})=\hat{I}$. We implemented three principal types of single-photon unital channels, with a controlled amount of noise, operating independently on both photons. The first channel is a one-field dephasing channel, where its single-qubit operation is described by

$$
\mathcal{E}(\hat{\rho})=(1-P) \hat{\rho}+P \sigma_{1} \hat{\rho} \sigma_{1} .
$$

$P$ is the probability to apply the channel, and $\sigma_{1}, \sigma_{2}$, and $\sigma_{3}$ are the Pauli matrices. The channel is composed of two translatable quartz wedges (see Fig. 10) that create a variable temporal delay between the polarization modes 35]. The second channel is a two-field channel

$$
\mathcal{E}(\hat{\rho})=(1-P) \hat{\rho}+\frac{P}{2} \sigma_{1} \hat{\rho} \sigma_{1}+\frac{P}{2} \sigma_{2} \hat{\rho} \sigma_{2},
$$



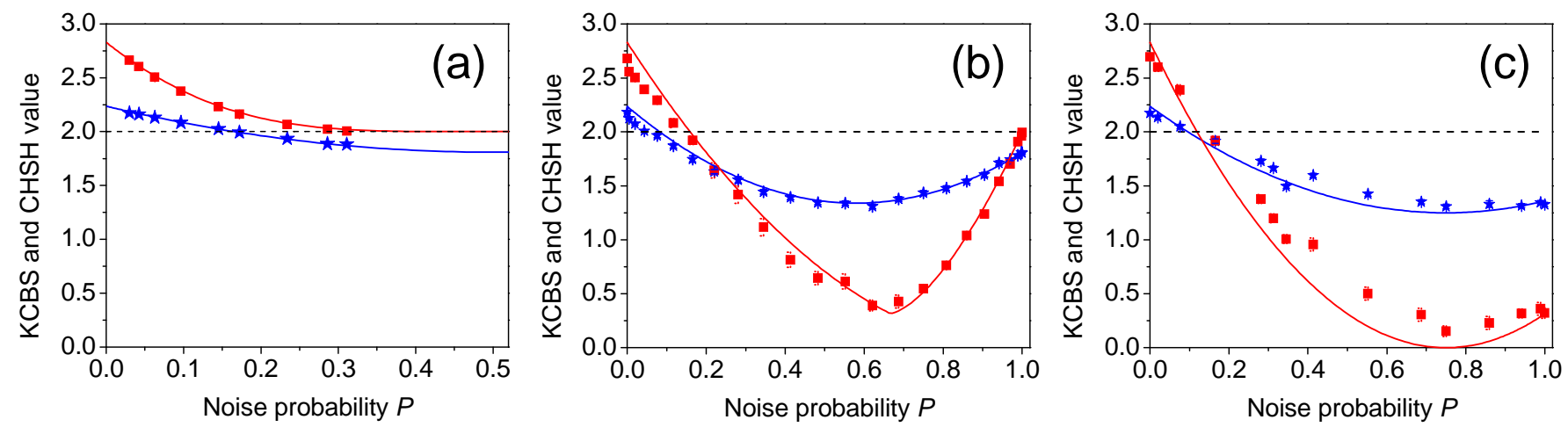

FIG. 2. (Color online) The KCBS and the CHSH values in the presence of unital noise. The maximal possible expectation values for the KCBS (blue pentagrams) and for the CHSH (red squares) operators of the output biphoton states are presented as a function of the noise probability. Data is presented for the three principal unital channels: (a) dephasing, (b) two-field and (c) isotropic channels. Solid lines represent the theoretical predictions. Dashed lines represent the value 2 - the upper bound allowed by any classical theory for the expectation values of both operators.

and the third one is an isotropic three-field channel

$$
\mathcal{E}(\hat{\rho})=(1-P) \hat{\rho}+\frac{P}{3} \sigma_{1} \hat{\rho} \sigma_{1}+\frac{P}{3} \sigma_{2} \hat{\rho} \sigma_{2}+\frac{P}{3} \sigma_{3} \hat{\rho} \sigma_{3} .
$$

The second and third channels are composed of a sequence of fixed birefringent calcite crystals and HWPs [28, 29] (see Figs. 1k and 1d, respectively). Each crystal entangles the polarization modes of a photon with its internal temporal degrees of freedom (DOFs). Decoherence occurs if the photon detection is insensitive to the temporal delays, practically averaging over these DOFs [28, 36]. For both channel types, control over the noise probability $P$ is achieved by rotating the corresponding HWPs to different angle settings [28] (for further elaboration see supplementary material [31]).

The KCBS and the CHSH values of the output biphoton state as a function of the noise parameter $P$ of the different channels are shown in Fig. 2, Solid lines represent the theoretical predictions for ideal $|\psi\rangle$ initial states. For the dephasing channel, the noise probability $P$ was deduced from the purity of the measured state (details in the methods section). For the two other channels, the noise probability $P$ was derived from the corresponding HWP settings. Thus the theoretical predictions for the two-field and the isotropic channels are without any fit parameters.

As can be seen by comparing the state dynamics of the different channels presented in Fig. 2, by adding more fields to the decoherence process, the KCBS and $\mathrm{CHSH}$ values can reach lower values. It is evident that for all three channels, the ability to violate the KCBS inequality is lost earlier than the ability to exhibit nonlocality. Specifically, for the dephasing channel (Fig. 2a), nonlocality is preserved for all noise probabilities (except for the extreme case of $P=0.5$ ), while the ability to exhibit KCBS correlations vanishes at $P \sim 0.17$. The results also demonstrate quantum hierarchy; a two-photon state that can exhibit KCBS correlations can also exhibit nonlocality, while the inverse is not true [26]. The deviation between measurement and theory for the isotropic channel (Fig. 22) is explained by the usage of narrower interference bandpass filters ( $3 \mathrm{~nm}$ instead of $5 \mathrm{~nm}$ ) that on one hand increased the fidelity of the initial state, but on the other, extended its coherence time. Since the noise channel operation relies on the finite coherence time of the incoming light, a longer coherence time reduces its depolarization strength. Nevertheless, The hierarchy described above holds also for the experimental results of this channel, as predicted.

In conclusion, we studied the ability of a symmetric two-photon state to exhibit quantumness. In the first part of the work, the KCBS value of a biphoton qutrit state was measured. The direct projection method was extended to the case where some of the biphoton population is distinguishable, and thus it resides also in the singlet subspace. The measured KCBS value is above the limit allowed by any classical theory. Assuming fair sampling and that the projections indeed represent compatible measurements [37], it has been shown that there exists no joint probability distribution that can explain the measurement outcomes of an experiment performed on the investigated qutrit state. In the second part of this work, we transmitted biphotons through three principal types of unital channels, and observed the degradation in the state ability to violate the KCBS and the $\mathrm{CHSH}$ inequalities as the noise level is increased. We demonstrated the predicted hierarchy between the two properties, i.e., KCBS contextuality implies nonlocality.

From a quantum information point of view, nonlocality is an essential resource for quantum computation tasks. Recently, there has been a growing interest in the study of quantum contextuality as a resource for quantum information applications 38 41]. Our results show that when the two photons of a biphoton experience a unital 
process, their nonlocal correlations are more robust than their KCBS contextual correlations. This leads to two possible future research directions. It is interesting to search for contextual based-on quantum protocols, that use different correlations from the KCBS correlations. Can these correlations be more robust against noise, even with respect to nonlocal based-on quantum protocols? Additionally, one can wonder if the observed fragility of the KCBS correlations hints that KCBS-based quantum technologies will exhibit superior performance to current entanglement-based approaches.

We thank the Israeli Ministry of Science and Technology for financial support and the Israeli Science Foundation for supporting this work under Grants 546/10 and $793 / 13$.

[1] S. Kochen and E. P. Specker, J. Math. Mech. 17, 59 (1967).

[2] J. S. Bell, Rev. Mod. Phys. 38, 447 (1966).

[3] A. Einstein, B. Podolsky, and N. Rosen, Phys. Rev. 47, 777 (1935).

[4] J. S. Bell, Physics 1, 195 (1964).

[5] J. F. Clauser, J. Horne, A. Shimony, and R. Holt, Phys. Rev. Lett. 23, 880 (1969).

[6] A. Aspect, J. Dalibard, and G. Roger, Phys. Rev. Lett. 49, 1804 (1982).

[7] M. A. Nielsen and I. L. Chuang, Quantum Computation and Quantum Information (Cambridge University Press, Cambridge, U.K., 2000).

[8] R. Horodecki, P. Horodecki, and M. Horodecki, Phys. Lett. A 200, 340 (1995).

[9] N. Bohr, Phys. Rev 48, 696 (1935).

[10] A. Cabello and G. García-Alcaine, Phys. Rev. Lett. 80, 1797 (1998).

[11] C. Simon, M. Żukowski, H. Weinfurter, and A. Zeilinger, Phys. Rev. Lett. 85, 1783 (2000).

[12] A. Cabello, Phys. Rev. Lett. 101, 210401 (2008).

[13] R. Wright, in Mathematical Foundations of Quantum Mechanics, edited by A. R. Marlow, (Academic Press, San Diego, 1978), p. 255.

[14] A. A. Klyachko, M. A. Can, S. Binicioğlu, and A. S. Shumovsky, Phys. Rev. Lett. 101, 020403 (2008).

[15] Y.-F. Huang, C.-F. Li, Y.-S. Zhang, J.-W. Pan, and G.C. Guo, Phys. Rev. Lett. 90, 250401 (2003).

[16] B. H. Liu, Y. F. Huang, Y. X. Gong, F. W. Sun, Y. S. Zhang, C. F. Li, and G. C. Guo, Phys. Rev. A 80, 044101 (2009).

[17] E. Amselem, M. Rådmark, M. Bourennane, and A. Cabello, Phys. Rev. Lett. 103, 160405 (2009).

[18] Y. Hasegawa, R. Loidl, G. Badurek, M. Baron, and H. Rauch, Nature 425, 45 (2003).

[19] G. Kirchmair et al., Nature 460, 494 (2009).

[20] O. Moussa, C. A. Ryan, D. G. Cory, and R. Laflamme, Phys. Rev. Lett. 104, 160501 (2010).

[21] R. Lapkiewicz, P. Li, C. Schaeff, N. K. Langford, S. Ramelow, M. Wieśniak, and A. Zeilinger, Nature 474, 490 (2011).

[22] M. Um et al., Sci. Rep. 3, 1627 (2013).
[23] J. Ahrens, E. Amselem, A. Cabello, and M. Bourennane, Sci. Rep. 3, 2170 (2013).

[24] Xi. Kong et al., arXiv:1210.0961v1.

[25] A. V. Burlakov, M. V. Chekhova, O. A. Karabutova, D. N. Klyshko, and S. P. Kulik, Phys. Rev. A 60, 4209 (1999).

[26] A. Soeda, P. Kurzyński, R. Ramanathan, K. Modi, and D. Kaszlikowski, Phys. Rev. A 87, 022120 (2013).

[27] R. B. A. Adamson, L. K. Shalm, M. W. Mitchell, and A. M. Steinberg, Phys. Rev. Lett. 98, 043601 (2007).

[28] A. Shaham and H. S. Eisenberg, Phys. Rev. A 83, 022303 (2011).

[29] A. Shaham and H. S. Eisenberg, Opt. Lett. 37, 2643 (2012).

[30] C. K. Hong, Z. Y. Ou, and L. Mandel, Phys. Rev. Lett. 59, 2044 (1987).

[31] See supplemental material for an elaboration on the experimental setup, the implementation of the unital channels, and for the projection settings for the KCBS measurements.

[32] M. V. Chekhova, L. A. Krivitsky, S. P. Kulik, and G. A. Maslennikov, Phys. Rev. A 70, 053801 (2004).

[33] D. F. V. James, P. G. Kwiat, W. J. Munro, and A. G. White, Phys. Rev. A 64, 052312 (2001).

[34] J. B. Altepeter, E. R. Jeffrey, and P. G. Kwiat, Adv. At. Mol. Opt. Phys. 52, 105 (2005).

[35] D. Branning, A. L. Migdall, and A. V. Sergienko, Phys. Rev. A 62, 063808 (2000).

[36] P. G. Kwiat, A. J. Berglund, J. B. Altepeter, and A. G. White, Science 290, 498 (2000).

[37] O. Gühne, M. Kleinmann, A. Cabello, J. Å. Larsson, G. Kirchmair, F. Zähringer, R. Gerritsma, and C. F. Roos, Phys. Rev. A 81, 022121 (2010).

[38] K. Horodecki, M. Horodecki, P. Horodecki, R. Horodecki, M. Pawłowski, and M. Bourennane, arXiv:1006.0468 1.

[39] M. Howard, J. Wallman, V. Veitch, and J. Emerson, Nature 510, 351 (2014).

[40] Z. Gedik, arXiv:1403.5861v2.

[41] S. Dogra, Arvind, and K. Dorai, arXiv:1406.5026v1. 


\section{Supplementary Material}

\section{EXPERIMENTAL SETUP}

Biphotons are collinearly generated in the process of spontaneous parametric down conversion. Using a lens of $30 \mathrm{~cm}$ focal length (L1), a pulsed $390 \mathrm{~nm}$ pump laser was focused onto two perpendicularly oriented $1 \mathrm{~mm}$ thick type-I $\beta-\mathrm{BaB}_{2} \mathrm{O}_{4}$ (BBO) crystals. After the crystals, the down-converted signal is separated from the pump beam using a dichroic mirror (DM). A half-wave plate (HWP) denoted by $(\delta)$ is placed before the generating crystals in order to control the ratio between the pumping power of each crystal. Thus, the generated neutrally polarized state is $|\psi\rangle=\left(|2,0\rangle+e^{i \varphi}|0,2\rangle\right) / \sqrt{2}$. By tilting the temporal walk-off compensating crystal, which is placed after the generating crystals, the $\varphi$ angle is controlled. The state is filtered spatially using a single-mode fiber (SM), and spectrally by $3 \mathrm{~nm}$ or $5 \mathrm{~nm}$ interference bandpass filters (IF). The HWP denoted by $\alpha$ can rotate a $\left|\psi_{p m}\right\rangle$ state to the $|1,1\rangle$ state if $\alpha=22.5^{\circ}$. In the state tomography unit, the photons are split probabilistically at a beam splitter (BS). The required settings for the projection protocol and for the quantum state tomography procedure are achieved by a sequence of a quarter-wave-plate (QWP), a HWP, another QWP and a polarizing beam splitter (PBS), that are placed before the single-photon detectors of each port.

The quantum noise channels were placed before the BS. The noise probability of the dephasing channel is controlled by the translation of two quartz wedges in different directions, in order to change the optical length inside the birefringent wedges. The time delay range that can be set between the two polarizations is $\sim 380 \mathrm{fs}$. The measurements of Fig. 2a in the main text were taken through all this range. The noise probability $P$ was deduced from the purity $\left(\Pi=\operatorname{Tr}\left(\hat{\rho}^{2}\right)\right)$ of the measured state. Assuming that the initial state is ideal, that the channel is a dephasing channel, and that $P \leq 0.5$, we use the relation $P=\left(1-(2 \Pi-1)^{\frac{1}{4}}\right) / 2$ to obtain $P$. The noise probabilities of the two-field and the isotropic channels are controlled by the rotation of the corresponding channel middle wave-plate. For both channels $P=\sin ^{2}(2 \theta)$, where for the isotropic channel $\theta$ is $\theta_{2}$. The initial state for the one- and three-field channels was $\left|\psi_{p m}\right\rangle$. Due to technical considerations, a $\left|\psi_{r l}\right\rangle$ state was used for the two-field channel.

\section{PROJECTION SETTINGS FOR THE KCBS MEASUREMENTS}

The five $\left|l_{s t, k}\right\rangle$ states used in the projection protocol have the coefficients $\left\{\alpha_{0}, \alpha_{1}, \alpha_{2}\right\}_{k}=$ $\left\{\sin (\theta) / \sqrt{2}, \cos (\theta) e^{i \varphi_{k}},-\sin (\theta) e^{2 i \varphi_{k}} / \sqrt{2}\right\}, \quad$ where $\theta=\cos ^{-1}\left(\frac{1}{\sqrt[4]{5}}\right) \simeq 48.03^{\circ}$ for every $\left|l_{s t, k}\right\rangle$ and $\varphi_{k}=\frac{4}{5} \pi k$. These states are pairwise orthogonal $\left|l_{s t, k}\right\rangle \perp\left|l_{s t, k+1}\right\rangle$ $(k+1$ modulo 5$)$ and satisfy the maximal violation of Eq. (2) of the main text for an initial $|1,1\rangle$ state. The corresponding orthogonal single photon polarizations $\left|s_{k}\right\rangle$ and $\left|t_{k}\right\rangle$ that compose every $\left|l_{s t, k}\right\rangle$ state are presented in Fig. 3 Note that these 10 polarization states form two pentagrams with a symmetry axis that connects the $|h\rangle$ and $|v\rangle$ antipodes, which compose the $|1,1\rangle$ state. In order to project the $|1,1\rangle$ state onto all the $\left|s_{k}\right\rangle_{1}\left|t_{k}\right\rangle_{2}$ and $\left|t_{k}\right\rangle_{1}\left|s_{k}\right\rangle_{2}$ states, as required by the projection protocol, the three wave plates in both ports after the BS were tuned to the angles listed in table [I] Then, the coincidence counts of $|h\rangle_{1}|v\rangle_{2}\left(|v\rangle_{1}|h\rangle_{2}\right)$ detectors were measured, and the projection results of $\left|s_{k}\right\rangle_{1}\left|t_{k}\right\rangle_{2}\left(\left|t_{k}\right\rangle_{1}\left|s_{k}\right\rangle_{2}\right)$ were deduced.

\begin{tabular}{|l|r|r|c|c|c|}
\hline \multicolumn{1}{|c|}{$\mathrm{k}$} & 1 & 2 & 3 & 4 & 5 \\
\hline \hline QWP (a) & $3.92^{\circ}$ & $-9.91^{\circ}$ & $12.01^{\circ}$ & $-9.91^{\circ}$ & $3.92^{\circ}$ \\
\hline HWP & $-11.39^{\circ}$ & $+6.92^{\circ}$ & 0 & $-6.92^{\circ}$ & $11.39^{\circ}$ \\
\hline QWP (b) & $-3.92^{\circ}$ & $9.91^{\circ}$ & $-12.01^{\circ}$ & $9.91^{\circ}$ & $-3.92^{\circ}$ \\
\hline
\end{tabular}

TABLE I. The wave-plate angles for the direct projection measurements of the KCBS value. In order to successfully project a biphoton on a certain $\left|s_{k}\right\rangle_{1}\left|t_{k}\right\rangle_{2}$ or $\left|t_{k}\right\rangle_{1}\left|s_{k}\right\rangle_{2}$ state, each photon coming from the two ports of the BS has to pass through a QWP (a), a HWP, and another QWP (b), whose angles are shown in this table for every $k$. Then, the photons pass through a polarizer in each arm, oriented horizontally or vertically, where the orientation of the two polarizers is perpendicular, i.e., either $|h\rangle_{1}|v\rangle_{2}$ or $|v\rangle_{1}|h\rangle_{2}$.

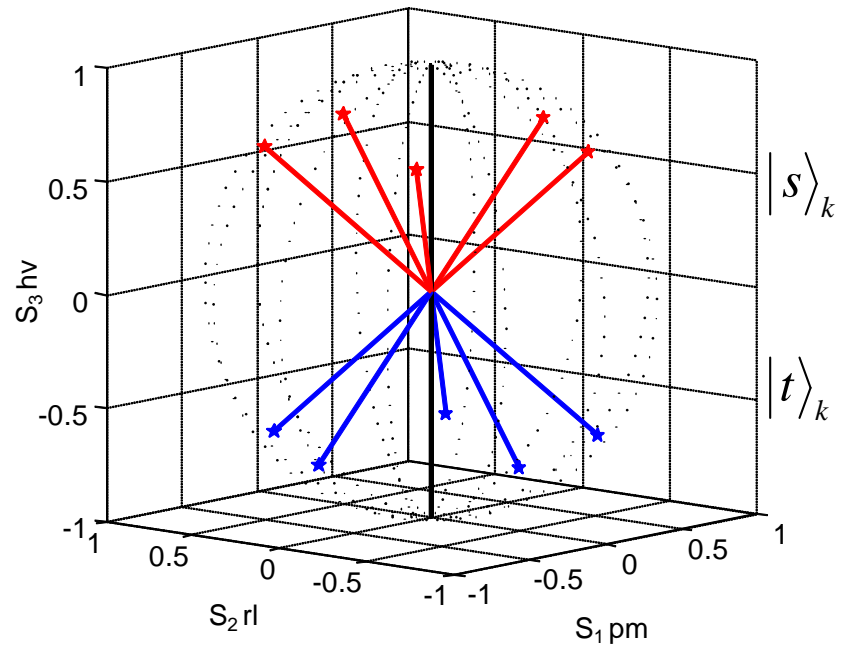

FIG. 3. (Color online) The polarization states used for the direct projection protocol. The $5\left|s_{k}\right\rangle$ states, along with their antipodes $\left|t_{k}\right\rangle$ states are presented in the Stokes representation. All 10 states have the same distance from the black solid line that connects the horizontal (h) and the vertical (v) polarizations, located at the poles of the Poincaré sphere. 